\title{
"Skin-out" Health Care Research: A Case for Community-Based Outcomes Studies
}

\author{
Robert Walker, MD ${ }^{1}$
}

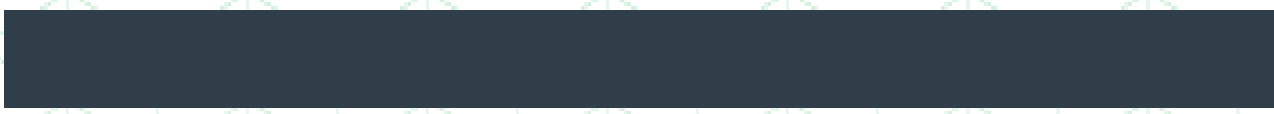

Author affiliations are

listed at the end of this article.

Correspondence to: Robert Walker, MD West Virginia Higher Education Policy Commission robert.walker@wvhepc.edu

Years ago, as a young zoology major at the University of Florida, I was faced with a major career decision. Although the major required the usual middle level chemistry, physics, and mathematics, the curriculum allowed alternative pathways. One option emphasized the cellular level of animals, with courses in biochemistry and cell biology. The other stressed natural history with courses in animal behavior and ecology. We affectionately called these two options "Skin-out" and "Skin-in." Students could prepare for a career in an application of laboratory science or for involvement in descriptive biology or wildlife management. My life took an entirely separate course, one I have never regretted, to medicine. But had I chosen a career in the biological sciences, it would have probably been the "Skin-out" option.

I would like to make a case for clinician directed, community-based research of the "Skin-out" variety. Such research does, of course, occur, and there is a degree of renewed interest. But the support for it pales in comparison to laboratory-based efforts. Laboratory-based research is critical to the advancement of the biomedical sciences. Support for this activity should and must continue. I would like to make a case for increased support for studies of the natural history of health and disease in the context of the community.

West Virginia communities, especially those that are underserved and disadvantaged, deserve to be studied. We assume, sometimes rather complacently, that biomedical research performed at urban academic health centers effectively translates into the lives of our rural citizens. This is often a safe assumption. But West Virginians are different. Health is not only rooted in our anatomy and physiology but in our culture, our chosen habits, occupations, and recreations and additionally, in our geology and geography. We need to know what works, and works well, for West Virginians as well as for people who live near academic health centers.

West Virginia's non-academic health providers deserve to be involved. Many, but not all, community-based health care providers are interested in organizing and analyzing their practice data in the context of the community. They find that such an activity is intellectually gratifying, provides an alternative to a busy practice routine, and offers ways 
to improve or reinforce care decisions. A lot of this activity is consistent with the quality improvement process required of federally qualified health centers. There is obvious potential for closer collaboration between community-providers, clinicians based at academic health centers, and state and local health departments.

Community-based, outcomes-oriented research deserves to be rigorous and receive reasonable support. Such research should not be merely a hobby or diversion for clinicians. This rigor requires refinement of the questions to be answered, precise definitions, well-planned data collection, and protection of the individuals and community to be studied. In my opinion, the traditional Institutional review process needs adaptation to communitybased research, but this is another discussion. Likewise, the process of collecting data for research purposes on one's own patients raises issues of ethics and bias that must be considered.

Tax-payers and policy-makers deserve more and higher quality studies of health outcomes in communities. Reimbursement for medical care has traditionally been provided based on process rather than outcome. As policy makers and citizens search for better value by paying for more favorable outcomes, we must find better ways to define and understand the term. Reasonable models for "pay for performance" have been proposed for ambulatory care and will need to be tested in rural populations. Marshall has a rich and productive history in community-based health care projects. The nation's first rural cancer prevention project, funded by the American Cancer Society and published in Cancer, was conducted jointly by Marshall and Lincoln Primary Care Center. The Benedum Rural Geriatrics Project, in which hundreds of rural, communityliving elders were followed for eight years, was formally presented to Congress and cited in the in the development of Medicare's current home-based waiver program. Projects based in rural communities and dealing with obese children were published in journals such as The American Journal of Clinical Nutrition before such topics were fashionable.

I would suggest that many questions involving health outcomes cannot be studied outside of the context of the community. West Virginia's academic health centers, in collaboration with motivated community-based clinicians are ideally situated to propose and answer these questions for central Appalachia and rural America. Such information will help us more effectively translate the care we are theoretically capable of providing into real care, for real patients, in real communities. The newest, and most productive and exciting research frontiers may not only involve new discoveries in the laboratory but new ways to describe and positively influence the "natural history" of health and disease in our patients.

\section{AUTHOR AFFILIATIONS}

1. Vice Chancellor for Health Sciences, West Virginia Higher Education Policy Commission 